\title{
PRONOSTICANDO EL ÍNDICE ENSO VARIOS PASOS EN ADELANTE MEDIANTE TÉCNICAS DE MODELAMIENTO NO LINEAL
}

\section{FORECASTING ENSO SEVERAL STEPS AHEAD THROUGH NONLINEAR MODELING TECHNIQUES}

\author{
Giovanni Salini Calderón ${ }^{1}$ \\ Recibido 25 de septiembre de 2009, aceptado 10 de noviembre de 2010 \\ Received: September 25, 2009 Accepted: November 10, 2010
}

\begin{abstract}
RESUMEN
Se indica cómo manejar una gran base de datos consistente de series temporales no lineales, aplicando distintas técnicas de modelamiento no lineal a estas series. Aunque no existen guías explícitas de manipulación de series temporales no lineales en la profusa bibliografía actual, existen diferentes enfoques que pueden ser tomados en cuenta. Para ello se estudió una base de datos mensual correspondiente a datos del Fenómeno del Niño (ENSO), entre los años 1866 y 2006. Se explica cómo debe manipularse esta base de datos que poseen características de no linealidad, la cual será usada para hacer pronósticos varios pasos en adelante. Se aplicaron dos test estándar: Información Mutua Promedio (AMI) y Falsos Vecinos más Cercanos (FNN). Se obtuvo el espaciamiento óptimo de los datos, así como el número de datos hacia atrás necesarios para pronosticar valores hacia el futuro. Luego se diseñaron varios modelos de redes neuronales artificiales (RNA), con diferentes reglas de aprendizajes, funciones de transferencia, elementos de procesamiento (o neuronas) en la capa escondida, etc., que permitieron hacer pronóstico de hasta 20 pasos en adelante. Las mejores redes correspondieron a aquellas que poseían como regla de aprendizaje la Regla Delta y la Regla Extendida, con función de transferencia sigmoide y tangente hiperbólica. El tipo de RNA usada fue una de multicapas alimentada hacia adelante y entrenada mediante la técnica de propagación hacia atrás. Se probaron redes con una, dos capas ocultas y sin ninguna capa. El mejor modelo que se obtuvo resultó ser uno consistente de una capa oculta.
\end{abstract}

Palabras clave: Series temporales no lineales, redes neuronales artificiales, pronóstico, dinámica no lineal, fenómeno del niño (ENSO), AMI, FNN.

\begin{abstract}
We indicate how to handle a large database consisting of nonlinear time series, applying different nonlinear modelling techniques to this kind of times series. Nowadays in the current references there is no explicit guide of how to manipulate data from nonlinear time series; however, there are approaches that can be taken account. To this end we studied a monthly database corresponding to South Oscillation Index (SOI) and between the years 1866 to 2006. It explains how there must manipulated this database whose data possess nonlinear characteristic, which will be used to do forecasts several steps ahead. Two standard tests to this database were applied, the Average Mutual Information (AMI) and the False Nearest Neighbours (FNN). The optimal spacing of the information was obtained as well as the number of values backward necessary to predict values towards the future. Then, several models were designed of artificial neural nets (ANN), with different learning rules, function of transfer, elements of process (or neurons) in the hidden layer, etc., that allowed to do forecasting of up to 20 steps ahead. The best networks were those that possessed the rules of learning called extDBD and Delta-Rule, and sigmoid as well as hyperbolic tangent as function of transfer. The type of used network was one of feedforward multilayer perceptron and trained by means of backpropagation technique. Networks were proved by one, two hidden layers and without any hidden layer. The best model that was obtained it turned out to be one that consisted with an alone hidden layer.
\end{abstract}

Keywords: Nonlinear times series, artificial neural networks, forecast, nonlinear dynamics, SOI, ENSO, AMI, FNN.

\footnotetext{
1 Departamento de Matemática y Física Aplicadas. Facultad de Ingeniería. Universidad Católica de la Ssma. Concepción. Fono: 41-2735549. Concepción, Chile.E-mail: gsalini@ucsc.cl
} 


\section{INTRODUCCIÓN}

A través de técnicas de modelamiento no lineal para sistemas dinámicos [1] se ha logrado caracterizar diferentes tipos de series temporales de carácter no lineal [2-3]. Para ello se emplean herramientas matemáticas que permiten determinar ciertos parámetros que entregan más luz en el análisis de una serie de tiempo no lineal. El primero de ellos se llama función AMI (información mutua promedio o average mutual information); y el segundo es la función FNN (falsos vecinos más cercanos ofalse nearest neighbours). Por otro lado, se han introducido como nueva técnica al estudio de las series temporales no lineales las denominadas Redes Neuronales Artificiales (RNA) [4], las cuales se han revelado como una herramienta capaz de modelar series temporales no lineales a las herramientas ya tradicionales [5-6].

La función AMI, I $(\tau)$, brinda el promedio de información binaria transmitida entre datos separados un intervalo de tiempo T, teniendo su propio mínimo en cierto instante $\tau$, correspondiente al espaciamiento implicado en una correlación entre datos sucesivos que entrega un mejor modelamiento [7]. Este primer mínimo se considera un buen estimador del tiempo para efectuar la reconstrucción del atractor en el espacio de las fases. Esta función se define como [1]:

$$
\begin{gathered}
l(\tau)= \\
\sum_{s(n), s(n+\tau)} P(s(n), s(n+\tau)) \log _{2}\left[\frac{(P(s(n), s(n+\tau))}{(P(s(n)) P(s(n+\tau)))}\right]
\end{gathered}
$$

donde $P(s(n))$ es la probabilidad de obtener el valor $s$ en el instante $\mathrm{n}, \mathrm{y}$ siendo $\mathrm{P}(\mathrm{s}(\mathrm{n}), \mathrm{s}(\mathrm{n}+\tau))$ la probabilidad conjunta de conseguir un valor dado de la variable $s$ en el instante $n+\tau$, dado el valor en el instante $n$.

Con la función FNN, es posible hallar la dimensión encajada $d_{e}(\tau)$, la cual define el número de valores previos de la serie que va a determinar el siguiente valor. En otras palabras, corresponde a la mínima cantidad de variables dinámicas necesaria para modelar nuestro sistema, que en el caso que nos interesa corresponde a la cantidad de datos pasados consecutivos que se requiere para hacer un buen pronóstico en un cierto intervalo de tiempo hacia el futuro. Sin embargo, si su curva decrece rápidamente, pero nunca va a cero, habría indicio cierto que se estaría en presencia de ruido.
Esta función se define como:

$$
\sqrt{\frac{R_{d+1}^{2}(k)-R_{d}^{2}(k)}{R_{d}^{2}(k)}}=\frac{\left|s(k+d \tau)-s^{N N}(k+d \tau)\right|}{R_{d}(k)}
$$

En esta expresión, $\mathrm{R}_{\mathrm{d}}{ }_{\mathrm{d}}$ es el cuadrado de la distancia euclidiana entre puntos vecinos más cercanos, vistos desde un espacio en $d$ dimensiones; y lo mismo para $\mathrm{R}_{\mathrm{d}+1}^{2}$.

La metodología RNA, por otro lado, es una metodología ampliamente difundida [8] y aplicada a un amplio espectro de áreas, como física, química, geofísica, geografía, contaminación ambiental, robótica, medicina, astronomía, ingeniería, finanzas, entre otras. Existen diversos estudios sobre bases de datos de series temporales no lineales relativos a fenómenos meteorológicos y atmosféricos [9], así como también a la contaminación ambiental [10-11], usando redes neuronales artificiales para hacer pronósticos ciertos pasos en adelante, con interesantes resultados. Una de las fortalezas que posee esta metodología es su tolerancia al fallo -la pérdida de unas pocas neuronas no afecta su funcionamiento. Además, estas redes pueden aprender y, lo más importante, tienen la capacidad de generalizar. Para ello, la base de datos debe subdividirse en subconjuntos consistentes de datos para la etapa de entrenamiento, de validación y otra para la etapa de test, para el caso de redes que requieren de un profesor (redes tipo supervisadas), como lo son las redes de multicapas.

En la modelación de una RNA se deben considerar las siguientes características: arquitectura de la red (disposición de los nodos o PE's, así como su número); tipo de conexión (simétrica o asimétrica); tipo de dinámica o algoritmo de actualización de la red (secuencial -o asincrónica-, paralela -o sincrónica-, estática o dinámica); regla de aprendizaje (supervisado o no supervisado) y tipo de función de transferencia (lineal, con umbral, no lineal).

Entre las redes más comúnmente usadas están las de tipo backpropagation o de multicapas (MLP) consistente de una capa de entrada, una o más capas ocultas y una capa de salida. Los PE's a determinar en la capa de entrada, en el caso de series temporales no lineales, es mediante las técnicas no lineales señaladas arriba. Los PE's de la capa escondida se determinan mediante el método experimental (de ensayo y error). Y los PE's de la capa de salida corresponden al pronóstico que se desea hacer, uno o varios pasos en adelante y puede haber uno o más nodos en esta capa. Este tipo de red, denominada también red de multicapas alimentada hacia adelante y con retropropagación como algoritmo de aprendizaje, 
aprende calculando un error entre la salida actual y la deseada, propagando este error de información hacia atrás a cada nodo de la red. La tasa de aprendizaje o coeficiente de aprendizaje, es la tasa a la cual este error modifica los pesos. El momentum es otro parámetro que también controla el aprendizaje, el cual dice que si los pesos son cambiados en cierta dirección, debiera de haber una tendencia para ellos a seguir cambiando en esa dirección. Así, una red de multicapas estándar puede aprender con distintas reglas de aprendizaje. Estas pueden ser la regla delta estándar, del tipo DBD (delta-bar-delta o regla delta), la regla delta extendida (extDBD o extended DBD), Quickprop, Maxprop, Fastprop, entre otras variantes de la primera. Debe considerarse también el tipo de función de transferencia a usar y que puede ser lineal, tanh, sigmoide, DNNA, etc., teniendo usualmente la siguiente forma:

$$
f(X)=\frac{1}{1+e^{-X}}
$$

El perceptrón, por su parte, consiste de una capa de entrada y una de salida, que al implementarse con una función de transferencia lineal puede decirse que es un método de regresión lineal en que todas las neuronas de la capa de entrada se conectan con una única (o más) neurona en la capa de salida. Sin embargo, la red de multicapas incorpora una función no lineal, siendo su argumento una combinación lineal de valores de la capa previa, que genera un valor final en las neuronas de la capa de salida, por lo que sirve de comparación respecto de los perceptrones.

La forma como se actualizan los pesos es mediante la siguiente ecuación (la cual es una variante del algoritmo estándar):

$$
\Delta \omega^{[s]}{ }_{j i}(t+1)=l \operatorname{coef} \times e^{[s]}{ }_{j} x^{[s-1]}+m o m \times \Delta \omega^{[s]}{ }_{j i}(t)
$$

Acá $\mathrm{X}^{[\mathrm{s}-1]}{ }_{\mathrm{i}}$ es la entrada de la neurona $i$ en la capa previa $(\mathrm{s}-1) ; \mathrm{e}^{[\mathrm{s}]}{ }_{\mathrm{j}}$ es el error local en la j-ésima neurona en el nivel $s$; lcoef es un coeficiente de aprendizaje; mom es el término de momentum que evita un lento aprendizaje, sirviendo para aumentar la velocidad de convergencia; $\Delta \omega^{[\mathrm{s}]}{ }_{\mathrm{ji}}$ se conoce como la regla del gradiente descendente para los pesos, uniendo a la i-ésima neurona en la capa (s-1) con la j-ésima neurona en la capa $s$. Esta ecuación es una variante del algoritmo estándar conocido. Este método, aunque un poco lento, es suficiente para el estudio de base de datos no lineales, requiriéndose más tiempo de computación.
Por su parte, el fenómeno de El Niño-La Niña (El Niño oscilación sur o ENSO), el cual involucra la elevación o descenso de la temperatura superficial del mar, ocurre en una escala de tiempo interanual, desarrollándose en una escala de miles de kilómetros cuadrados y con ello, influenciando el clima global. Nominado así por los pescadores de las costas del Perú y el Ecuador para referirse a la corriente de aguas más cálidas que típicamente aparece en la época de la navidad, durando varios meses y siendo los peces menos abundantes en este período. Se define como el promedio trimestral de la temperatura superficial del mar que se desplaza desde una región normal (Darwin) a una crítica del Pacífico ecuatorial (Tahití), la cual contiene una banda o corriente de agua fría que se extiende a lo largo del ecuador desde la costa sudamericana al océano Pacífico central. Estos corrimientos desde la temperatura media superficial del mar en esta región son críticamente importantes, pues determinan un mayor desplazamiento en el patrón de lluvia tropical, la cual a su vez influye en los patrones de temperatura y precipitación alrededor del mundo.

Para ser clasificado como un evento del Niño o la Niña, debe ser por un período de tiempo mayor a cinco meses consecutivos. Un parámetro histórico para caracterizar la magnitud del evento de El Niño-La Niña es mediante el índice de oscilación sur (SOI, o southern oscillation index). Este índice refleja la magnitud y fase de la componente atmosférica del ENSO. Valores negativos de este índice indican condiciones de El Niño, y valores positivos para La Niña. Un valor igual a cero corresponde a un caso normal [12].

El índice SOI se define como: normalizado en Darwin)

Es bien sabido [13] el impacto tanto climático como económico (daño en silvicultura, agropecuario, etc.) que ha estado produciendo este fenómeno meteorológico a nivel planetario. Por ello, sería bien interesante para la comunidad científica y la población contar con una herramienta que permita predecir cuándo aparecerá uno u otro fenómeno.

En la literatura actual hay pocos trabajos que abordan el estudio de este tipo de serie temporal y, aún más, con la perspectiva de hacer pronóstico no sólo uno sino varios pasos en adelante [14-16]. 
Este artículo está organizado de la siguiente manera. En la sección siguiente se explica la metodología empleada para el estudio de la base de datos del SOI. Se diseñan las redes neuronales que se usarán para hacer predicción ciertos pasos en adelante, determinándose para ello los parámetros más relevantes. En la siguiente sección se muestran los resultados obtenidos, al hacer pronóstico varios pasos en adelante para la serie del SOI a la cual se le aplicó primero un filtro pasa bajo (FPB) y luego un filtro pasa alto (FPA). Se comparan dos tipos de modelos de predicción: lineal y no lineal. Finalmente, en conclusiones se extraen los resultados más relevantes obtenidos en este estudio.

\section{DATOS Y SELECCIÓN DE PARÁMETROS}

Para entender mejor cómo aplicar la metodología de las técnicas de modelamiento no lineal a una base de datos muy compleja, es que se ha elegido aquella que dice relación con el Fenómeno del Niño y que consistió de datos mensuales del SOI entre los años 1866 y 2006, en total 1.692 datos.

La base de datos se procesó usando los valores obtenidos para los parámetros $\tau$ y $d_{e}$. Específicamente, se seleccionaron siete entradas (las mismas que la dimensión de encaje) y una única salida correspondiente al pronóstico de un paso en adelante (o más, como se verá más adelante), que se quiere efectuar.

Luego se procedió a adecuar la base de datos para ser usada en nuestra RNA, modificando la base de datos, desde una consistente inicialmente de una sola columna, a una que tuviese ocho columnas. Esto correspondería a la información a entregar a la RNA, consistente en siete columnas de datos para la capa de entrada y una columna como salida, correspondiente al dato a pronosticar. En total se generaron de este modo 212 datos (denominados también epoch), aproximadamente. Esta nueva base de datos se dividió a su vez en tres bases de datos: una para entrenar la red, consistente de 122 datos; la otra base de datos para validar la red consistió de 50 datos; y la última consistió de 40 datos para test de nuestro modelo. Con esto, se reducían a 320 meses, aproximadamente, los datos a ser pronosticados que corresponden a los últimos 26 años (vale decir, el período comprendido entre 1980 y 2006).

Por su parte, a la base de datos original (1.692 datos) se le aplicaron dos filtros: un filtro pasa bajo (FPB) y un filtro pasa alto (FPA), quedando con 1.691 datos cada una de ellas. A continuación se le aplicó la metodología no lineal a estas nuevas series temporales, obteniéndose una dimensión de encaje de cinco para la base FPB y de ocho para la FPA. Al acomodar estas bases para la etapa de entrenamiento, se obtuvieron 1.687 y1.684 datos, respectivamente. La primera base (FPB) se subdividió, a su vez, en una de entrenamiento (1.012 datos), una para validación (387 datos) y una de test (337 datos), con cinco PE's de entrada y un PE de salida. Por su parte, la segunda base (FPA) se subdividió, a su vez, en una de entrenamiento (1010 datos), una para validación (337 datos) y una de test (337 datos), con ocho PE's de entrada y un PE de salida.

Estas mismas bases de datos se modificaron para hacer pronóstico de hasta 20 pasos en adelante, lo cual hizo variar la cantidad de datos en las bases original para entrenamiento, validación y test. Cabe hacer notar que en un trabajo anterior (Salini [10]), con estas mismas metodología sólo se hace un pronóstico de un paso en adelante. Luego, los diferentes pronósticos, para varios pasos en adelante, efectuados en este trabajo demostrarán la potencialidad del método RNA.

Se diseñaron dos tipos de redes neuronales: un perceptrón y una red MLP. Las características de cada una de ellas se describen en la siguiente sección.

\section{ANÁLISIS Y RESULTADOS}

De acuerdo a la Figura 1, la función AMI muestra que el primer mínimo ocurre para un tiempo de rezago de 10 , para la base de datos original (serie mensual El Niño). La Figura 2 muestra la función FNN, obteniéndose una dimensión de encaje de siete, para esta misma base de datos.

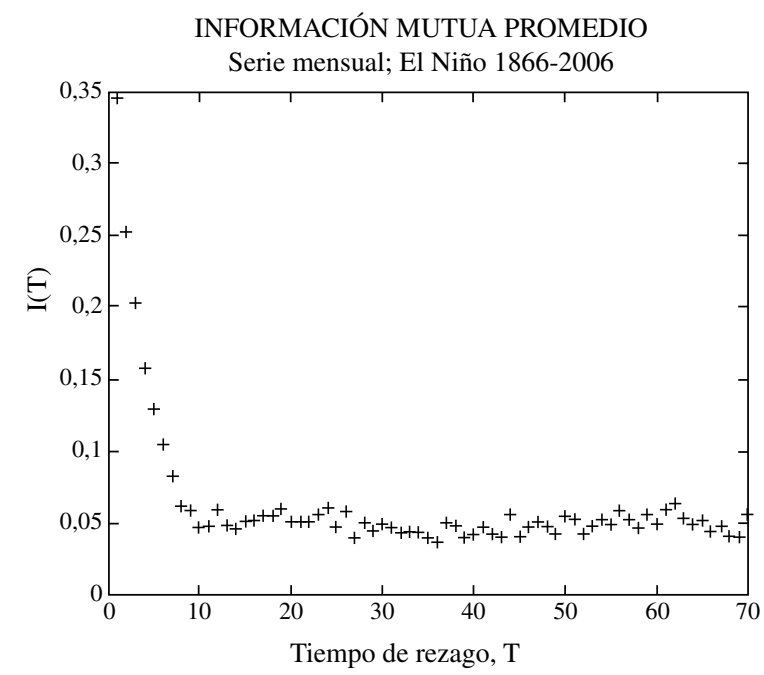

Figura 1. Información mutua promedio para la serie mensual El Niño 1866-2006. 


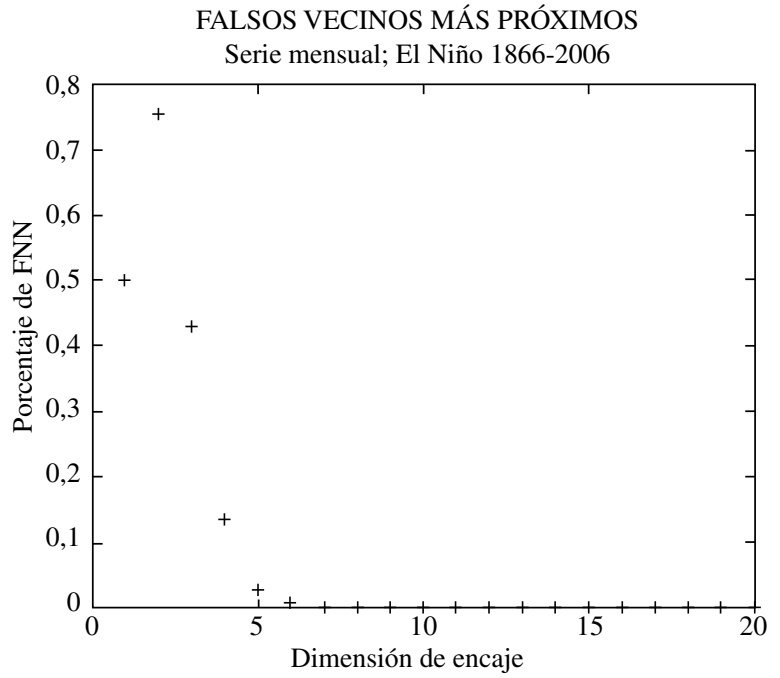

Figura 2. Porcentaje de falsos vecinos más cercanos para la serie mensual El Niño 1866-2006.

Como criterio de comparación de los modelos se usó el error de la raíz cuadrática promedio normalizado, RMSE - N (por root mean square error normalized), y definido por la ecuación (6), en que $O$ corresponde al valor real y $P$ al predicho.

$$
\mathrm{RMSE}-\mathrm{N}=\frac{\sqrt{\frac{1}{N} \sum_{i}\left(O_{i}-P_{i}\right)^{2}}}{\frac{\sum_{i} O_{i}}{N}}
$$

El error en la predicción se calculó, entonces, comparando el valor predicho por la red en la etapa de test, con el valor que efectivamente se presentó. Como función correlación, se usó la definición estándar.

Se diseñó, a continuación, un perceptrón con una capa de entrada (de siete PE's) y una capa de salida de un PE, correspondiente al valor a pronosticar.

Posteriormente se diseñó una nueva red tipo MLP para la base de datos mensual, cuya arquitectura se muestra en la Figura 3, para la base de datos original. Para diseñar esta red se aplicó el método de aprendizaje conocido como backpropagation, cuya arquitectura definitiva consistió de una capa de entrada con siete neuronas, una o dos capas escondidas con el número de PE's a ser determinado (usualmente mediante ensayo y error) y una capa de salida, con un PE de salida -correspondiente al valor a ser pronosticado- para uno o más pasos en adelante en el pronóstico. Por lo que lo único que quedó por determinar fueron los PE's de la capa escondida.

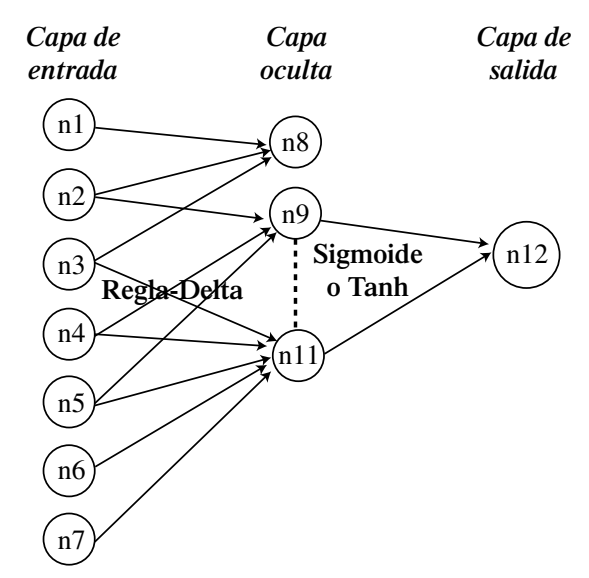

Figura 3. Arquitectura de una red MLP. Se muestran algunas conexiones entre los nodos de entrada y la capa oculta y entre esta última y el PE de salida.

La Tabla 1 muestra el valor RMSE-N para los dos modelos de RNA aplicados a los 212 datos estudiados. En esta tabla se presentan los parámetros usados para las diferentes redes neuronales implementadas. Se aprecia que la "mejor" red neuronal consistió de una capa escondida y un error RMSE-N de 0,8397. La peor red neuronal fue una de dos capas escondidas y un RMSE-N de 0,9142.

Tabla 1. Valores RMSE-N para dos tipos de modelos de red neuronal.

\begin{tabular}{|r|r|l|l|l|l|}
\hline NNCO & Mom & $\begin{array}{c}\text { R } \\
\text { Apren }\end{array}$ & \multicolumn{1}{|c|}{ TF Trans } & $\begin{array}{c}\text { R } \\
\text { Lcoef }\end{array}$ & RNME-N \\
\hline \multicolumn{7}{|c|}{ Perceptrón simple } \\
\hline 0 & 0,4 & D-R & L & 0,3 & 0,8864 \\
\hline 0 & 0,4 & D-R & T & 0,3 & 0,8877 \\
\hline \multicolumn{7}{|c|}{ Red de Multicapas (MLP) } \\
\hline 1 & 0,4 & D-R & S & 0,3 & 0,8741 \\
\hline 1 & 0,6 & D-R & S & 0,3 & 0,8623 \\
\hline 1 & 0,4 & D-R & T & 0,3 & 0,8397 \\
\hline $1^{*}$ & 0,4 & D-R & S & 0,3 & 0,8666 \\
\hline $2^{* *}$ & 0,4 & D-R & S & 0,3 & 0,8606 \\
\hline 2 & 0,4 & D-R & S & 0,3 & 0,9142 \\
\hline 2 & 0,4 & D-R & T & 0,3 & 0,8903 \\
\hline 3 & 0,7 & D-R & S & 0,3 & 0,8848 \\
\hline
\end{tabular}

L: Lineal; S: Sigmoide; T: Tanh

NNCO: número de neuronas en capa oculta.

Mom: valor del término de momentum.

R Apren: regla de aprendizaje.

TF Trans: tipo de función de transferencia.

R LCoef: razón del coeficiente de aprendizaje.

RMSE-N: error de la raíz cuadrática promedio normalizado.

$\mathrm{N}-\mathrm{C}$ : regla de aprendizaje acumulativa normalizada.

D-R: regla delta generalizada.

*: Red de dos capas ocultas con un PE cada una.

**: Red de dos capas ocultas con 2 PE's cada una. 
En resumen, podemos observar de esta tabla que las redes MLP con una capa escondida, con función de transferencia S o T, son las que mejor aprendieron. A su vez, las redes multicapas con dos o tres capas escondidas no consiguieron mejor aprendizaje.

En el caso de los perceptrones, tuvieron un peor desempeño que las MLP con una capa escondida, pero mejor que con dos o tres capas escondidas.

En la Figura 4 se muestran dos casos de pronóstico con un paso en adelante, para cada tipo de modelo implementado, en base a los datos de la Tabla 1.

A continuación se procedió a determinar el RMSE-N y la correlación existente entre el objetivo y el valor predicho, tanto para la base de datos FPB como para la FPA. En la Figura 5 se muestran dos casos de pronóstico de un paso
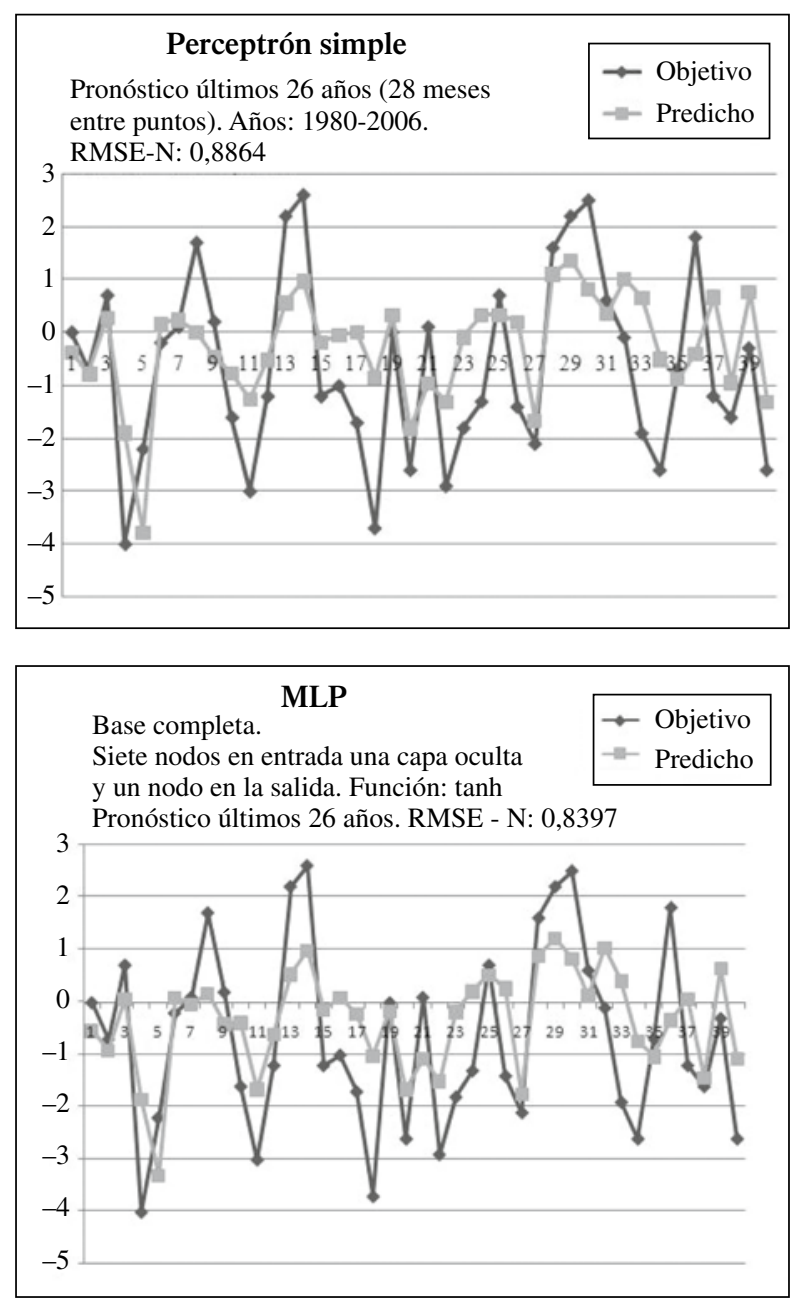

Figura 4. Pronóstico un paso en delante de una red perceptrón y una MLP, últimos 26 años. en adelante y cuatro pasos en adelante, para la base de datos FPB. En el primer caso, su RMSE-N fue de 0,0167 y un valor de correlación (entre el objetivo y el predicho) de 0,9877. En el segundo caso, el RMSE-N fue de 0,1016, con un valor de correlación de 0,9320.

\section{PRONÓSTICO UN PASO ADELANTE}

Capa oculta: 1 PE; función sigmoide;

RMSE-N: 0,0167. Correlación: 0,9877. Base de Datos: FPB (años 1978-2006)
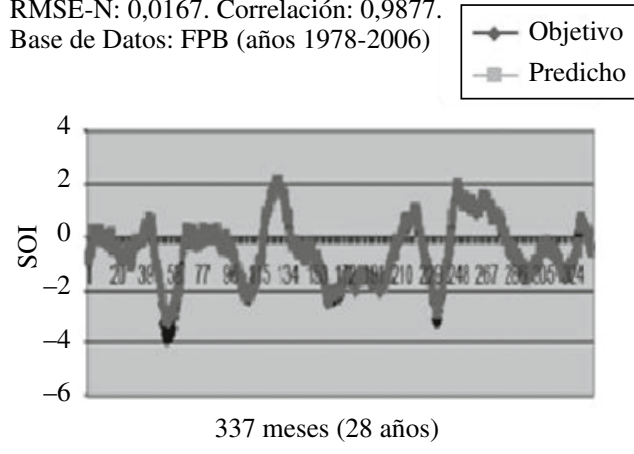

\section{PRONÓSTICO CUATRO PASOS EN ADELANTE}

Capa oculta: 1 PE; función sigmoide;

RMSE-N: 0,1016. Correlación: 0,9320.

Base de Datos: FPB (años 1978-2006)
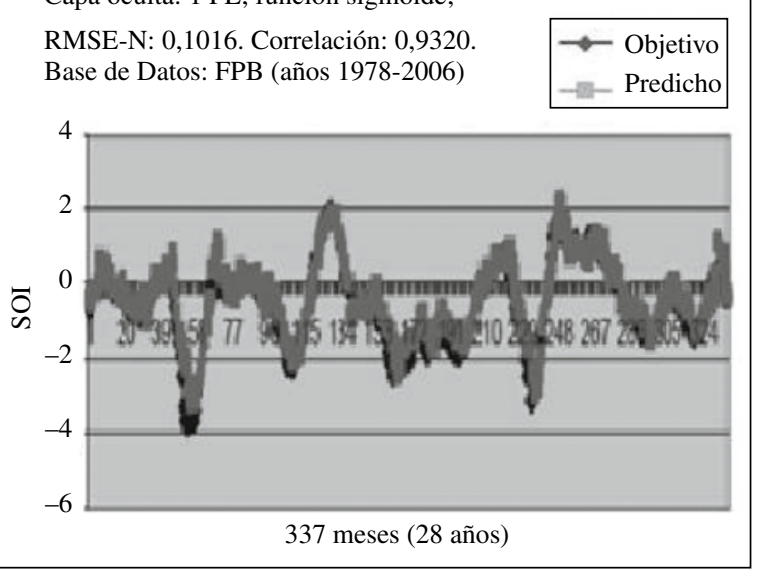

Figura 5. Pronóstico de uno y cuatro pasos en delante de una red MLP, últimos 28 años.

En la Tabla 2 se pueden observar los valores de correlación y RMSE-N para la base de datos SOI con filtro pasa bajo, FPB, hasta 20 pasos en adelante como pronóstico. La mejor arquitectura para esta base de datos resultó ser una red neuronal con una capa escondida.

Se puede observar de esta tabla que la mejor red resultó ser extDBD con un $70 \%$ de correlación hasta siete pasos en adelante. Sin embargo, puede observarse, además, que en diez pasos en delante de pronóstico, aún es bueno su valor de correlación (62\%). La peor red neuronal resultó ser DeltaDBD, con función de transferencia tanh. Por otro 
lado, en la capa oculta se probaron -mediante ensayo y error-, hasta diez PE's, resultando satisfactorio cuatro y siete PE's. Cabe hacer notar que diez PE's no lograron mejorar notablemente la capacidad de pronóstico de la red neuronal.

Tabla 2. Pronóstico SOI filtro pasa bajo, desde uno hasta 20 pasos en adelante.

Tabla de correlaciones entre serie original (objetivo) y la predicha

\begin{tabular}{|c|c|c|c|c|c|c|c|c|}
\hline \multirow{6}{*}{$\begin{array}{l}1 \text { Paso } \\
\text { adelante }\end{array}$} & \multirow{3}{*}{$\begin{array}{l}N^{0} \text { PE's } \\
1 \text { Capa } \\
\text { Escond. }\end{array}$} & \multicolumn{7}{|c|}{ Regla de Aprendizaje } \\
\hline & & \multirow{2}{*}{$\begin{array}{c}\text { exDBD } \\
\text { Tanh }\end{array}$} & \multicolumn{3}{|c|}{ Regla Delta } & \multicolumn{2}{|c|}{ Delta DBD } & \multirow{2}{*}{$\begin{array}{l}\text { Quick-Prop } \\
\text { Sigmoide }\end{array}$} \\
\hline & & & Sigmoide & Tanh & Sigmoide & Tanh & Tanh & \\
\hline & 4 & 0.6194 & 0,9868 & 0,9915 & 0,9845 & 0,9403 & 0,0000 & 0,9788 \\
\hline & 5 & 0,9890 & 0,9891 & 0,9868 & 0,9845 & 0,9336 & 0,3867 & 0,9743 \\
\hline & 6 & 0,9579 & 0,9895 & 0,9870 & 0,9861 & 0,9339 & 0,6179 & 0,9691 \\
\hline & 2 & 0,5512 & 0,9749 & 0,9704 & 0,9680 & 0,8734 & 0,6343 & 0,9670 \\
\hline 2 Pasos & 4 & 0,9376 & 0,9755 & 0,9715 & 0,9685 & 0,9044 & 0,7279 & 0,9621 \\
\hline adelante & 6 & 0,9403 & 0,9754 & 0,9721 & 0,9699 & 0,8915 & 0,8941 & 0,9510 \\
\hline & 7 & 0,9742 & 0,9750 & 0,9726 & 0,9693 & 0,8548 & 0,7149 & 0,9474 \\
\hline & 4 & 0,6194 & 0,9897 & 0,9864 & 0,9845 & 0,9403 & 0,0000 & 0,9788 \\
\hline & 5 & 0,9890 & 0,9891 & 0,9868 & 0,0845 & 0,9336 & 0,3867 & 0,9743 \\
\hline & 6 & 0,9579 & 0,9895 & 0,9870 & 0,9861 & 0,9339 & 0,6179 & 0,9691 \\
\hline & 2 & 0,8696 & 0,9121 & 0,9104 & 0,9039 & 0,7357 & 0,2369 & 0,9005 \\
\hline elante & 5 & 0,9320 & 0,9114 & 0,9110 & 0,9001 & 0,7542 & 0,0111 & 0,8866 \\
\hline & 7 & 0,9111 & 0,9243 & 0,9119 & 0,9026 & 0,7119 & 0,6830 & 0,8765 \\
\hline 5 Pasos & 2 & 0,7734 & 0,8655 & 0,8654 & 0,8558 & 0,6709 & 0,6790 & 0,8515 \\
\hline adelante & 7 & 0,8704 & 0,8643 & 0,8671 & 0,8543 & 0,6117 & 0,8356 & 0,8646 \\
\hline & 1 & 0,0000 & 0,8097 & 0,8075 & 0,7913 & 0,5429 & 0,6401 & 0,7835 \\
\hline adelante & 2 & 0,8102 & 0,8082 & 0,8112 & 0,7974 & 0,5359 & 0,1577 & 0,7914 \\
\hline & 7 & 0,8164 & 0,8070 & 0,8144 & 0,7952 & 0,5027 & 0,3191 & 0,7592 \\
\hline & 1 & 0,0000 & 0,7422 & 0,7453 & 0,7442 & 0,3838 & 0,3574 & 0,6983 \\
\hline adelante & 2 & 0,5792 & 0,7411 & 0,7457 & 0,7286 & 0,4011 & 0,5801 & 0,7212 \\
\hline & 7 & 0,7460 & 0,7399 & 0,7451 & 0,7262 & 0,3843 & 0,4166 & 0,6782 \\
\hline & 1 & 0,0000 & 0,6645 & 0,6754 & 0,6454 & 0,1932 & 0,0055 & 0,5991 \\
\hline asos & 2 & 0,6698 & 0,6645 & 0,6688 & 0,6490 & 0,2764 & 0,0536 & 0,6398 \\
\hline & 4 & 0,6648 & 0,6636 & 0,6685 & 0,6457 & 0,4815 & 0,3464 & 0,6300 \\
\hline & 2 & 0,5484 & 0,5821 & 0,5831 & 0,5620 & 0,1672 & $-0,0437$ & 0,5473 \\
\hline adelante & 3 & 0,58 & 0,5816 & 0,5848 & 0 & 0,1771 & 0,1160 & 0,5288 \\
\hline & 4 & 0,6242 & 0,5811 & 0,5837 & 0,5590 & 0,3509 & $-0,0001$ & 0,5383 \\
\hline & 4 & 0,5044 & 0,4988 & 0,4979 & 0,4656 & 0,2488 & 0,0853 & 0,6208 \\
\hline adelante & 5 & 0,5050 & 0,6091 & 0,4988 & 0,5866 & 0,1539 & $-0,098$ & 0,3727 \\
\hline & 6 & 0,5030 & 0,4959 & 0,4973 & 0,4675 & 0,1476 & 0,4413 & 0,5594 \\
\hline asos & 4 & 0,0005 & 0,4072 & 0,4062 & 0,3662 & 0,1539 & $-0,0011$ & ),3169 \\
\hline adelante & 5 & 0,4338 & 0,4100 & 4099 & & 09 & 88 & 2299 \\
\hline & 10 & 0,4000 & $0,4\left(\begin{array}{lll}3 \\
0\end{array}\right.$ & 0,4092 & 0,375 & 0,0611 & 0,1219 & 0,1465 \\
\hline 12 Pasos & 2 & 0,0000 & 0,3155 & 0,3141 & 2689 & 0,0047 & 0,0000 & 0,0688 \\
\hline adela & 4 & 0,3210 & 0,3108 & 0,3121 & 0,2679 & 0,1063 & 0,0036 & 0,2078 \\
\hline & 10 & 0,3073 & 0,3039 & 0,3125 & 0,2758 & 0,0351 & 0,1116 & 0,0811 \\
\hline 14 Pasos & 1 & 0,1783 & 0,1450 & 0,1194 & 0,1360 & 0,0367 & 0,0000 & 0,0638 \\
\hline adelante & 6 & 0 & 0,1492 & 1315 & & 37 & 0,0019 & 0,0656 \\
\hline & & & 0,1448 & 0,1264 & & 0,0257 & 0,1002 & 0,0555 \\
\hline $16 \mathrm{P}$ & 3 & 0,0852 & 0,0886 & 0,0538 & 0 , & 0,0 & 0,0003 & 0,0657 \\
\hline adelante & 4 & 0,0000 & 0,0877 & 0,0586 & 0,0882 & 0,0788 & 0,0004 & 0,0776 \\
\hline & 6 & 0,0329 & 0,0962 & 0,0597 & 0,0912 & 0,0712 & $-0,0686$ & 0,0731 \\
\hline 18 Pasos & 4 & 0,0222 & 0,0659 & 0,04 & 0,0769 & 0,0842 & 0,0505 & 0,0806 \\
\hline adelante & 6 & 0,02 & 0,0819 & 0,04 & 0,080 & 0, & 0,0918 & 0,0810 \\
\hline & 10 & & & 0,04 & & 0,0081 & 0,2369 & 0,0776 \\
\hline & 1 & 0,0000 & 0 , & 0,0367 & & 0,0845 & 0,0140 & \\
\hline d & 2 & 0,06 & 0,0511 & 0,0328 & 0,0672 & 0,0704 & 0,0603 & 0,0783 \\
\hline & 6 & 0,0317 & 0,0791 & 0,0324 & 0,0798 & 0,824 & 0,1955 & 0,0817 \\
\hline & & & lores & & - Indi & & is alto & \\
\hline & & & & & In & & más & \\
\hline & & & & & : Valor & obal má & to de cor & ción \\
\hline
\end{tabular}

Finalmente, en la Tabla 3 se presenta una tabla de valores de correlación y RMSE-N para la base de datos FPA, filtro pasa alto. A pesar de que se repitió el mismo tipo de cálculo usado para la base FPB, sólo se incluyeron acá los resultados de hasta tres pasos en adelante, debido a que los pronósticos para más pasos en adelante fueron muy bajos en su correlación y no aportaron más información.

Puede observarse que de este tipo de red la mejor resultó ser QuickProp, con un 60\% aproximadamente, de correlación un paso en adelante. Sin embargo, puede observarse, además, que tres pasos en delante de pronóstico empeoran el valor de correlación.

La peor RNA resultó ser Norm-Cum-Delta, con función de transferencia sigmoide. Por otro lado, en la capa oculta se probaron -mediante ensayo y error-, hasta diez PE's, resultando satisfactorio hasta siete PE's. Cabe hacer notar que con diez PE's no logró mejorar notablemente la capacidad de pronóstico de la red neuronal.

En un estudio posterior de esta base de datos se determinará la predictibilidad bajo el supuesto de que haya cierta caoticidad en ella, para poder determinar hasta con cuántos pasos en adelante puede hacerse un pronóstico confiable.

Tabla 3. Pronóstico SOI filtro pasa alto, desde uno hasta tres pasos en adelante.

$$
\begin{aligned}
& \text { TABLA DE CORRECCIONES } \\
& \mathbf{N}^{\circ} \\
& \text { PE's } \\
& \text { Tipo Regla de Aprendizaje }
\end{aligned}
$$
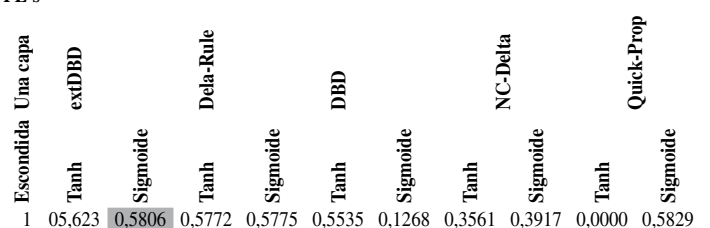

$\begin{array}{llll}0.623 & 0.5806 & 0.5772 & 0,5775\end{array}$ $\begin{array}{lllllllllllll}2 & 0,5599 & 0,5818 & 0,5744 & 0,5825 & 0,5533 & 0,1492 & 0,5688 & 0,4962 & 0,0552 & 0,5926\end{array}$ $\begin{array}{llllllllllll}1 \text { Paso } & 3 & 0,5795 & 0,5771 & 0,5796 & 0,5772 & 0,4792 & -0,1199 & 0,4824 & 0,3803 & 0,0084 & 0,5773\end{array}$

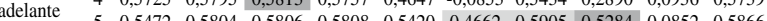
$\begin{array}{llllllllllll}5 & 0,5472 & 0,5804 & 0,5806 & 0,5808 & 0,5420 & 0,4662 & 0,5905 & 0,5284 & 0,0852 & 0,5866\end{array}$ $\begin{array}{lllllllllllll}6 & 0,5815 & 0,5796 & 0,5818 & 0,5806 & 0,5611 & 0,1785 & 0,3973 & 0,5100 & 0,0520 & 0,5900\end{array}$ $\begin{array}{llllllllllll}7 & 0,5305 & 0,5806 & 0,5818 & 0,5814 & 0,5735 & 0,4653 & 0,4801 & 0,5777 & 0,2322 & 0,5913\end{array}$

\begin{tabular}{llllllllllll}
1 & 0,1575 & 0,5031 & 0,5027 & 0,5001 & 0,4584 & $-0,0145$ & 0,1454 & 0,2334 & 0,0000 & 0,5003 \\
\hline
\end{tabular} $\begin{array}{llllllllllll}2 & -0,0357 & 0,5030 & 0,4974 & 0,5071 & 0,4384 & 0,1934 & 0,4276 & 0,4062 & 0,0185 & 0,4984\end{array}$ $\begin{array}{lllllllllllll}3 & 0,0187 & 0,4990 & 0,5026 & 0,5002 & 0,3798 & -0,1016 & 0,4000 & 0,2566 & 0,0251 & 0,4886\end{array}$ $\begin{array}{llllllllllllll}2 \text { Pasos } & 3 & 0,0187 & 0,490 & 0,5026 & 0,5002 & 0,3798 & -0,1016 & 0,4006 & 0,2566 & 0,0251 & 0,4886 \\ & 4 & 0,4748 & 0,4991 & 0,5035 & 0,4980 & 0,3519 & -0,0066 & 0,4360 & 0,1967 & 0,0013 & 0,4803\end{array}$

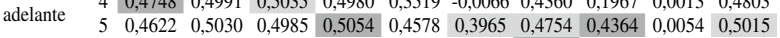
$\begin{array}{llllllllllll}5 & 0,4622 & 0,5030 & 0,4985 & 0,5054 & 0,4578 & 0,3965 & 0,4754 & 0,4364 & 0,0054 & 0,5015 \\ 6 & 0,4350 & 0,5005 & 0,5006 & 0027 & 0,4561 & 0,1597 & 0,4393 & 0,3944 & 0,1036 & 0,4975\end{array}$ $\begin{array}{llllllllllllll}7 & 0,4897 & 0,5405 & 0,5034 & 0,5040 & 0,4782 & 0,3768 & 0,3675 & 0,4694 & 0,0045 & 0,4974\end{array}$

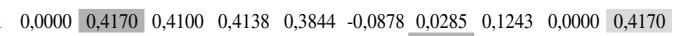
$2 \begin{array}{lllllllllllll}2,0000 & 0,4145 & 0,4145 & 0,4141 & 0,3052 & 0,1916 & 0,3666 & 0,2936 & 0,0785 & 0,3885\end{array}$ $\begin{array}{lllllllllll}3 & 0,0467 & 0,4109 & 0,4132 & 0,4123 & 0,3249 & -0,0626 & 0,2653 & 0,1956 & 0,0376 & 0,3965\end{array}$ $\begin{array}{llllllllllllll}3 \text { Pasos } & 4 & -0,0120 & 0,4121 & 0,4135 & 0,4057 & 0,2652 & 0,0467 & 0,3728 & 0,1760 & 0,1323 & 0,3862\end{array}$ $\begin{array}{llllllllllllll}\text { adelante } & 5 & 0,3147 & 0,4180 & 0,4148 & 0,4166 & 0,3260 & 0,2636 & 0,3240 & 0,3135 & 0,0298 & 0,3977\end{array}$ $\begin{array}{lllllllllllll}6 & 0,3852 & 0,4147 & 0,4153 & 0,4180 & 0,4142 & 0,1816 & 0,2232 & 0,3461 & 0,0299 & 0,4146\end{array}$ $\begin{array}{llllllllllll}6 & 0,3852 & 0,4147 & 0,4153 & 0,4180 & 0,4142 & 0,1816 & 0,2232 & 0,3461 & 0,0299 & 0,4146 \\ 7 & 0,3378 & 0,4164 & 0,4149 & 0,4174 & 0,4161 & 0,3431 & 0,2219 & 0,3836 & -0,0232 & 0,4040\end{array}$ Código colores

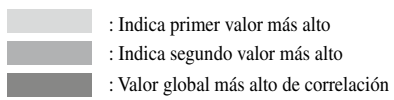

De la Tabla 3 se observó que la regla de aprendizaje que mejor funcionó fue extDBD (con función de transferencia 
sigmoide). La peor fue delta bar delta (DBD) con función de transferencia sigmoide en todas las etapas. Tener diez PE's en una capa escondida no mejora significativamente el aprendizaje ni rendimiento de la red. Las mejores de aprendizaje resultaron ser: extDBD y delta-rule.

\section{CONCLUSIONES}

Se observó que el perceptrón simple resultó ser una buena herramienta para hacer pronósticos para unos pocos pasos en adelante.

A pesar de que los perceptrones lineales realizaban buena predicción, las redes de multicapas (MLP) resultaron, en todos los casos, ser las que mejor predecían, en esta primera etapa de pronóstico, de un paso en adelante.

Con la metodología de las redes de multicapas diseñadas se obtiene una automatización satisfactoria del proceso de predicción de un paso en adelante de la serie temporal.

Además, se puede afirmar que cuando los efectos no lineales no son demasiado importantes en la modelación, las redes de multicapas no son significativamente mejores que el perceptrón. Sin embargo, como ocurrió en este caso, cuando estos efectos no lineales pasan a ser importantes las redes de multicapas son mejores en cuanto a su capacidad de predicción respecto de los modelos lineales.

Cuando se filtra la señal de la serie SOI, separándose las componentes pasa-bajo y pasa-alto, en el primer caso, se mejora bastante la predicción varios pasos en adelante. No obstante, en el segundo caso la predicción empeora a medida que nos alejamos varios pasos en adelante en el pronóstico.

Se observó que la regla de aprendizaje que mejor funcionó fue extDBD con función de transferencia sigmoide. La peor fue delta bar delta (DBD) con función de transferencia tanh en todas las etapas.

Por su parte, tener diez PE's en una capa escondida no mejora significativamente el aprendizaje ni rendimiento de la red.

En general, las mejores reglas de aprendizaje resultaron ser: extDBD y delta-rule.

Cuando se aplicó a la serie temporal original un filtro pasa bajo, y se efectuaron pronósticos de hasta veinte pasos en adelante, se observó que hasta para diez pasos en adelante el pronóstico fue bueno. Sin embargo, cuando se aplicó un filtro pasa alto a los mismos datos, sólo para unos pocos pasos en adelante el pronóstico resultó ser bueno.
Finalmente podemos afirmar que la predicción del índice SOI puede llevarse a cabo empleando técnicas utilizadas para modelar sistemas dinámicos, así como la técnica de las RNA.

\section{AGRADECIMIENTOS}

El autor agradece al profesor Dr. Rodrigo Abarca de la Universidad de Concepción por facilitar la base de datos del SOI entre los años 1866 a 2006.

\section{REFERENCIAS}

[1] H. Abarbanel. "Analysis of Observed Chaotic Data". Institute for Nonlinear Science. SpringerVerlag. First edition, p. 272. New York, USA. 1996.

[2] M. Small. "Applied Nonlinear Time Series Analysis: Applications in Physics, Physiology and Finance". Word Scientific Series in Nonlinear Science. Serie A, World Scientific Publishing Company. First edition, p. 245. March 28, 2005.

[3] H. Kantz and T. Schreiber. "Nonlinear Time Series Analysis”. Cambridge University Press, Second edition, p. 370. Cambridge, UK, 2004.

[4] Y. H. Hu and J.N. Hwang (editors). "Handbook of Neural Network Signal Processing". CRC Press. First edition, p. 384. Boca Raton, FL., USA. 2002.

[5] G.E.P. Box, G.M. Jenkins and G.C. Reinsel. "Time Series Analysis; Forecasting and Control”. PrenticeHall. Third edition, p. 75. Englewood Cliffs, San Francisco, CA, USA. 1994.

[6] J. Hamilton. "Time Series Analysis". Princeton University Press, First edition, p. 799. Princeton, New Jersey, USA. 1994.

[7] A.M. Fraser and H.L. Swinney. "Independent coordinates for strange attractors from mutual information". Physical Review A. Vol. 33, pp. 11341140. February, 1986.

[8] K. Swingler. "Applying Neural Networks. A practical guide". Academic Press Limited. First edition, p. 320. San Diego, CA, USA. 1996.

[9] B. Hewitson and R. Crane. "Neural Nets: Applications in Geography”. Geo Journal Library. Kluwer Academic Publishers. First edition, p. 194. Dordrecht, The Netherlands. 1994.

[10] P. Pérez, A. Trier and J. Reyes. "Prediction of PM2.5 concentrations several hours in advance using neural networks in Santiago, Chile". Atmospheric Environment. Vol. 34, Issue 8, pp. 1189-1196. 2000 . 
[11] G. Salini and P. Pérez. "Estudio de series temporales de contaminación ambiental mediante técnicas de redes neuronales artificiales". Ingeniare. Revista Chilena de Ingeniería. Vol. 14 No 3, pp. 284-290. Septiembre-diciembre, 2006.

[12] C.F. Ropelewski and M.S. Halpert. "Precipitation patterns associated with high index phase of Southern Oscillation". Journal Climate. Vol. 2, pp. 268-284. 1989.

[13] C.F. Ropelewski and M.S. Halpert. "Global and regional scale precipitation pattern associated with El Niño/Southern Oscillation". Monthly Weather Review. Vol. 115, Issue 8, pp. 1606-1626. August, 1987.
[14] D. Korecha and A. Baruston. "Predictability of June-September Rainfall in Etiopía". Monthly Weather Review Vol. 135, Issue 2, pp. 628-650. February, 2007.

[15] N. Singhrattna, B. Rajagopalan, M. Clark and K. Kumar. "Seasonal forecasting of Thailand summer monsoon rainfall". International Journal of Climatology. Vol. 25, Issue 5, pp. 649-664. 2005.

[16] M.S. Baawin, M.H. Nour, A.G. El-Din and M. Gamal El-Din. "El Niño southern-oscillation prediction using southern oscillation index and Niño3 as onset indicators: Application of artificial neural networks". J. Environ. Eng. Sci. Vol. 4, Issue 2, pp. 113-121. 2005. 